\title{
CONTEMPORARY DIAGNOSTIC AIDS IN ENDODONTICS
}

Divya Jindal ${ }^{1}$, Deepak Raisingani², Medhavi Sharma ${ }^{3}$, Dileep Soni ${ }^{4}$, Hanisha Dabas ${ }^{5}$

\section{HOW TO CITE THIS ARTICLE:}

Divya Jindal, Deepak Raisingani, Medhavi Sharma, Dileep Soni, Hanisha Dabas. "Contemporary Diagnostic AIDS in Endodontics". Journal of Evolution of Medical and Dental Sciences 2014; Vol. 3, Issue 06, February 10; Page: 1526-1535, DOI: 10.14260/jemds/2014/2019

ABSTRACT: Management of any problem starts with an accurate diagnosis and this is not different in the field of endodontics. The diagnosis of dental pulp status should be seen as a synthesis of history, clinical examination, special tests and radiological examination, and not as the outcome of any one specific test. With the advent of technologies in our day to day life we need the help of technologies in dentistry also. An effort has been made to provide readers with contemporary, evidence based prospective on endodontic diagnosis. Vitality testing is an important aid in the diagnosis of pulp disease and apical periodontitis. The ideal pulp test method should provide a simple, objective, standardized, reproducible, nonpainful, noninjurious, accurate and inexpensive way of assessing the condition of the pulp tissue. This article describes the various technologies that are available to aid the dental practitioner in detecting a tooth with a relevant endodontic disease. It also explains the need and limitation of the technologies as an aid in our management of endodontically affected tooth.

KEYWORDS: Laser Doppler flowmetry, cone beam computed tomography, pulp oximetry.

INTRODUCTION: Diagnosis in dentistry may be defined as 'the process whereby the data obtained from questioning, examining and testing are combined by the dentist to identify deviations from the normal.1,2 The diagnosis of dental pulp status should be seen as a synthesis of history, clinical examination, special tests, and radiological examination, and not as the outcome of any one specific test $^{3}$. This article describes the various technologies available to aid the need and the importance of these technologies.

New image reconstruction techniques have been introduced that provide information threedimensionally to the clinician for routine endodontic and surgical treatment planning. The age of three-dimensional imaging and image processing is here.

LASER DOPPLER FLOWMETRY: This is a non invasive, objective, painless, semi-quantitative method, which is more reliable in measuring the blood flow to the pulp. Laser light is transmitted to the pulp by means of a fiber optic probe ${ }^{4}$. Laser Doppler flowmetry uses Helium Neon (HeNe) and Gallium Aluminum (Ga AlAs) as semiconductor diode lasers at a power of 1 to $2 \mathrm{~mW}$. The wave length of the HeNe laser is $632.8 \mathrm{~nm}$ and that of the semiconductor diode laser is 780 to $820 \mathrm{~nm}^{5}$. The ideal position to place the probe is 2 to $3 \mathrm{~mm}$ from the gingival margin ${ }^{6}$. The scattered light from the moving red blood cells in the circulation will be frequency-shifted, while those from the static tissues remain unshifted. The reflected light composed of Doppler shifted and unshifted light is returned by the afferent fibers and a signal is produced ${ }^{7}$.

The end of the LDF which contacts the tooth contains both sending and receiving optic fibers, with one of the configuration being one source and two detectors in a triangular arrangement at the probe end ${ }^{8}$. Calibration of the probes is important to ensure accurate readings ${ }^{9}$. The larger the optical fiber separation distance on the probe, the higher the signal output as a larger surface area is 
covered, and also there is potentially a higher chance of blood flow signal contamination of the nonpulp sources ${ }^{10}$. This technique can be successfully employed for estimating the vitality of the pulp in both adults and children.

Laser Doppler flowmetry is not useful in teeth with crowns and large restorations. The disadvantages of LDFs are that they detect only the coronal blood flow of the pulp, which may not relate to the actual blood flow on the linear scale. On the other hand advantages are that it allows painless diagnosis as compared to thermal \& electric pulp tests. It is useful for the vital or non vital diagnosis of immature or traumatized teeth, not meant for use in patients who are sensitive to tooth pain ${ }^{11,12}$. [Figure 1]

PULP OXIMETRY: The term 'oximetry' is defined as the determination of the percentage of oxygen saturation of the circulating arterial blood ${ }^{13}$. Pulse oximetry is a relatively inexpensive procedure which is commonly used in anesthetic procedures ${ }^{14,15}$. Pulse oximetry readily differentiates between vital and non vital teeth. Oxygenated hemoglobin and deoxygenated hemoglobin are different in color and therefore absorb different amounts of red and infrared light. The pulse oximeter therefore utilizes probes which emit red and infrared light to transilluminate the targeted vascular area, which allows the photo detectors to identify the absorbance peak due to a pulsatile blood circulation and thereby calculate the pulse rate and oxygen saturation levels ${ }^{13,15}$.

A pulse oximeter works on the principle that uses a photo electric diode that transmits light in two wave lengths. The probe is placed on the labial surface of the tooth crown and the sensor on the palatal surface. Ideal placement of the probe is in the middle third of the crown. If placed in the gingival third, disturbances from gingival circulation or any gingival trauma or bleeding will interfere with the readings. Useful in cases of impact injuries where blood supply remains intact but the nerve supply is damaged. Pulpal circulation can be detected independent of gingival circulation. Limitations include background absorption associated with venous blood and tissue constituents, which should be differentiated. This enables monitoring of the changes in oxygen saturation, thus detecting pulpal inflammation in the teeth that are still vital ${ }^{16}$.

Dual Wavelength Spectrophotometry: Dual wavelength spectrophotometry (DWLS) is a method independent of a pulsatile circulation. The presence of arterioles rather than arteries in the pulp and its rigid encapsulation by surrounding dentine and enamel make it difficult to detect a pulse in the pulp space. This method measures oxygenation changes in the capillary bed rather than in the supply vessels and hence does not depend on a pulsatile blood flow. DWLS detects the presence or absence of oxygenated blood at $760 \mathrm{~nm}$ and $850 \mathrm{~nm}$.

The blood volume or concentration channel is arranged to respond linearly to the increase in light absorption. The oxygenation channel senses the oxygenated blood because of the greater absorption at $850 \mathrm{~nm}$ as compared to $760 \mathrm{~nm}$. A major advantage is that it uses visible light that is filtered and guided to the tooth by fibreoptics ${ }^{17}$. [Figure 2]

CONE BEAM COMPUTED TOMOGRAPHY: This was initially developed for angiography in 1982. Principal feature of CBCT is that multiple planar projections are acquired by rotational scan to produce a volumetric dataset from which inter-relational images can be generated. Cone beam techniques mostly use a single rotation of $180^{\circ}$ or more in which the x-ray source and a reciprocating 
x-ray detector are attached by an arm and rotate around the patient's head [Figure 3]. The FOV determined by the area of interest is primarily dependent on the detector size, beam projection geometry, and selected collimation where available. Applications include evaluating the position of impacted teeth, supernumerary teeth, maxillary sinus position, mandibular canals and lingual nerves. This method is also useful in Implant planning, surgical assessment of pathology and preoperative/postoperative assessment of craniofacial fractures, evaluation of root fractures and trauma, analysis of external and internal root resorption and extraradicular invasive cervical resorption. Has higher resolution and diagnostic potential, focused on anatomical area of interest with less radiation exposure and less time required to read the image ${ }^{18}$.[Figure 4]

DIAGNODENT LASER SYSTEM: This is a laser fluorescence system that detects changes in the tooth structure due to demineralization. These structural changes cause an increase in the fluorescence at specific excitation wavelengths. The intensity of the fluorescence depends upon the wavelength of the light as well as the structure and condition of hard dentinal tissues. Diagnodent with a laser diode that generates a pulsed $655 \mathrm{~nm}$ laser beam via a central fiber is transported to the tip of the device and into the tooth. When the incident light interacts with tooth substance, it stimulates fluorescent or luminescent light at longer stokes shiftedwavelengths. The intensity of fluorescence is a function of the degree of demineralization or bacterial concentration in the probed region. The Diagnodent operates on the premise that a high level bacteria reading indicates a probability of having a decalcified enamel structure. Hence, one weakness of this technology is that all bacteria, not only caries-related bacteria, produce fluorescence. Advantages of the system are that the quantitative nature of its readings gives a basic guideline as to when to intervene. It is pertinent to mention that factors such as the presence of bacterial plaque, dental prophylactic pastes, fissure sealants, and composite resin materials give false positive readings with this instrument ${ }^{19}$. [Figure 5]

Hughes probeye camera: It can detect temperature changes as small as $0.10 \mathrm{C}$. It can also measure pulp vitality, by measuring blood flow in pulp. This consists of thermal video system with a silicon close up lens with a resolvable spot size of 0.023 inch.

Technique: teeth in question are isolated with rubber dam and cooled with stream of cold air. Symmetrical cooling of teeth of about $220 \mathrm{C}$ is done. Then teeth are rewarmed to their former temperature. Vital teeth will rewarm in 5 sec whereas non-vital teeth take up $15 \mathrm{sec}$ to rewarm. More rapid warming of vital teeth is due to an intact blood supply.

Tuned aperture computed tomography (TACT): Tuned aperture computed tomography works on the basis of tomosynthesis (Webber \& Messura 1999). A series of 8-10 radiographic images are exposed at different projection geometries using a programmable imaging unit, with specialized software to reconstruct a three-dimensional data set which may be viewed slice by slice.

Claimed advantages of TACT are that the images produced have less superimposition of anatomical noise over the area of interest (Webber et al. 1996, Tyndall et al.1997). The overall radiation dose of TACT is no greater than 1-2 times that of a conventional periapical X-ray film as the total exposure dose is divided amongst the series of exposures taken with TACT. Additional advantages claimed for this technique include the absence of artefacts resulting from radiation interaction with metallic restorations. Recently, studies have concluded that TACT is suitable for 
detecting vertical root fractures Tuned aperture computed tomography appears to be a promising radiographic technique for the future ${ }^{18}$.

Computed tomography (CT): Computed tomography is an imaging technique which produces three-dimensional images of an object by taking a series of two-dimensional sectional X-ray images. Essentially, CT scanners consist of a gantry which contains the rotating X-ray tube head and reciprocal detectors. In the centre of the gantry, there is a circular aperture, through which the patient is advanced. The tube head and reciprocal detectors within the gantry either rotate synchronously around the patient, or the detectors take the form of a continuous ring around the patient and only the X-ray source moves within the gantry. The data from the detectors produce an attenuation profile of the particular slice of the body being examined. The patient is then moved slightly further into the gantry for the next slice data to be acquired. The process is repeated until the area of interest has been scanned fully.

Current CT scanners are called multislice CT (MSCT) scanners and have a linear array of multiple detectors, allowing 'multiple slices' to be taken simultaneously, as the X-ray source and detectors within the gantry rotate around the patient who is simultaneously advanced through the gantry. This results in faster scan times and therefore a reduced radiation exposure to the patient. The buccolingual thickness of the cortical and cancellous bone, the position and inclination of the root within the mandible could only be assessed using CT. They concluded that CT should be considered before the surgical treatment of mandibular premolars and molars when on the dental radiograph the mandibular canal is not visible or in close proximity to the lesion/root. Gain additional information on the root canal anatomy and its relationship to vital structures such as the maxillary sinus using reconstructed axial slices and three-dimensional reconstruction of CT data. Computed tomography may also be useful for the diagnosis of poorly localized odontogenic pain. The assessment of the 'third dimension' with CT imaging also allows the number of roots and root canals to be determined, as well as where root canals join or divide. This knowledge is extremely useful when diagnosing and managing failing endodontic treatment. The uptake of CT in endodontics has been slow for several reasons, including the high effective dose and relatively low resolution of this imaging technique. Other disadvantages of CT are the high costs of the scans, scatter because of metallic objects, poor resolution compared with conventional radiographs and the fact that these machines are only found in dedicated radiography units. Access may thus be problematic for dentists in practice. CT technology has now become superseded by cone beam computed tomography (CBCT) technology in the management of endodontic problems ${ }^{18}$.

Potential endodontic applications include

1. Diagnosis of endodontic pathosis and canal morphology,

2. Assessment of pathosis of non-endodontic origin,

3. Evaluation of root fractures and trauma,

4. Analysis of external and internal root resorption and invasive cervical resorption,

5. Presurgical planning,

6. Treatment of aberrant and extra root canals,

7. Developmental anomalies like dens invaginatus, C-shaped canals, volumetric analysis and more. [Figure 6] 
Fiber-optic transillumination: Fiber-optic transillumination (FOTI) as a caries detection technique is based on the fact that carious enamel has a lower index of light transmission than sound enamel 20. The light is absorbed more when the demineralization process disrupts the crystalline structure of enamel and dentin. In essence this gives that area a more darkened appearance. This method of caries detection uses a light source; prefer-ably bright, to illuminate the tooth. Caries or demineralized areas in dentin or enamel show up as darkened areas with this technique. This effect can be achieved with a fiber optic illuminator, which is readily available at the hand piece coupler of the dental operatory and has been used for detection of approximal and occlusal caries.

Posterior approximal caries can be diagnosed with the light probe positioned on the gingivae below the cervical margin of the tooth, whereby the light passes through the tooth structures and approximal decay produces a dark shadow on the occlusal surface. Although this device has the advantage that the examination is done with an operating light source already available in general practice, it is only useful for approximal and occlusal lesions; its sensitivity and specificity are not sufficient for detection of very early caries ${ }^{21}$.

Digital imaging fiber-optic transillumination: This is a digitized and computed version of the FOTI. While FOTI was designed for detection of approximal and occlusal caries, digital imaging fiber-optic transillumination DIFOTI is used for detection of both incipient and frank caries in all tooth surfaces. DIFOTI can also be used to detect fractures, cracks, and secondary caries around restorations. DIFOTI uses white light to transilluminate each tooth and to instantly create high-resolution digital images of the tooth. It is based on the principle that carious tooth tissue scatters and absorbs more light than surrounding healthy tissue. Decay near the imaged surface appears as a darker area against the more translucent brighter background of surrounding healthy anatomy. A single fiber-optics illuminator in the mouthpiece delivers light to one of the tooth's surfaces. As this light travels through layers of enamel and dentin, it scatters in all directions toward the nonilluminated surface usually the opposite surface. The light is then directed through the mouthpiece to a miniature electronic charge coupled device CCD camera in the hand piece. The camera digitally images the light emerging from either the smooth surface opposite the illuminated surface or the occlusal surface. These images are displayed on a computer monitor in real time and stored on the hard drive for easy retrieval for comparative review of images over time. Image acquisition is controlled with software and a foot pedal.

Images of the teeth can be viewed by both the clinician and patient, and therefore can be used for patient education and motivation. It is important to note that DIFOTI images the light emerging from surface closest to the CCD camera. It does not image the tooth material between the light source and the CCD camera, and therefore cannot indicate the depth of lesion penetration ${ }^{21-27 .}$

Electrical caries monitor: This technology is based on the electrical conductivity differences between sound and carious dental tissues. ECM is based on the principle that electrical conductivity is a function of porosity. Enamel demineralization results to increased porosity of the enamel tissue and saliva fills the pores and forms conductive pathways for electrical transmission. Since saliva is a better electrical conductor than enamel tissue, the conductivity increases with demineralization. On this basis, ECM measures the electrical resistance of a site on the tooth during controlled drying. By drying the tooth surface, the resistance is determined by the tooth structure, avoiding electrical conductance by surface liquid saliva. 
High measurements indicate well-mineralized tissue while low values indicate demineralized tissue. The electrical conductivity of a tooth changes with demineralization even when the surface remains macroscopically intact. Example of this device is the lode electronic caries monitor. Conductivity is measured from the probe tip in the fissure through the dental pulp to a handheld earth lead with the patient forming part of the circuit. A major advantage of the ECM is to present objective readings, which have the potential for monitoring lesion progression, arrest, or remineralization.

The sensitivity and the specificity of this machine have been reported to be very high, 0.75 and 0.77 , respectively, when used to detect occlusal caries in vivo and ex vivo, indicating that it is a valid indicator for detecting the presence or absence of lesion porosity. A strong relationship between both lesion depth and mineral content in enamel has been shown with ECM readings. The only drawback is the fact that it is time consuming to use in a routine full-mouth examination ${ }^{28}$.

CONCLUSION: While some of the devices are fully developed and commercially available, others are still under development. The devices vary in their modes of action as well as their capability as caries diagnostic aids. It is clear that the differences in caries presentation and behavior in different anatomical sites make it unlikely that any one diagnostic modality will have adequate sensitivity and specificity of detection of carious lesions for all sites; a combination of diagnostic tools will help us diagnose lesions earlier and detect failing restorations sooner, all to avoid more costly, destructive dental procedures and truly take dentistry into the preventive rather than the reactive mode.

An accurate assessment of tooth vitality is of paramount importance in clinical practice. Rapid advances in knowledge and applied technology relating to pulpal blood flow may lead the way for a more objective, accurate, and predictable means of pulp vitality assessment. This article has provided a comprehensive review on the different approaches to pulp vitality assessment \& imaging techniques.

\section{REFERENCES:}

1. Baiju Gopalan nair, amarendhar reddy K; "A Review of Laser Doppler Flowmetry and Pulse Oximetry in Dental Pulp Vitality" Journal of Clinical and Diagnostic Research. 2011 August, Vol5(4): 903-905.

2. Robinson HB. The nature of the diagnostic process. Dent Clin North Am 1963; 1: 3-8.

3. Velayutham gopikrishna; Assessment of pulp vitality: a review; International Journal of Paediatric Dentistry 2009; 19: 3-15

4. Karayilmaz H, Kirzioglu Z; Comparison of the reliability of Laser Doppler Flowmetry, Pulse Oximetry and Electric Pulp Tester in assessing the vitality of human teeth." J Oral Rehabil. 2011 May;38(5): 340-7.

5. Matsumoto K. Lasers in Endodontics. Dental Clinics of North America 2000; 44/4: 889-906.

6. Vongsavan N, Matthews B. Experiments in pigs on the sources of laser Doppler blood flow signals which are recorded from teeth. Archives of Oral Biology 1996; 41/1: 97-103.

7. Ramsay D S, Artun J, Martinen S S. Reliability of pulpal blood-flow measurements which utilize laser Doppler flowmetry. Journal of Dental Research 1991; 70/11: 1427-1430.

8. Ingolfsson A R, Tronstad L, Hersh E V, Riva C E; Efficacy of laser Doppler flowmetry in determining the pulp vitality of human teeth. Endodontics and Dental Traumatology 1994; 10/2: 83-87. 
9. Roeykens H, Van Maele G; Reliability of laser Doppler flowmetry in a 2-probe assessment of pulpal blood flow; Oral Surgery, Oral medicine, Oral pathology, Oral radiology, and Endodontics 1999; 87/6: 742-748.

10. Ingolfsson A R, Tronstad L, Hersh E V, Riva C E. The effect of probe design on the suitability of Laser Doppler flowmetry in the vitality testing of human teeth." Endodontics and Dental Traumatology 1993; 9/2: 65-70.

11. Gazelius B, Lindh-Stromberg U, Pettersson H, Oberg P A. Laser Doppler technique - a future diagnostic tool for tooth pulp vitality. International Endodontic Journal 1993; 26/1: 8-9.

12. Polat S, Er K, Polat N T. Penetration depth of the Laser Doppler flowmetry beam in teeth. Oral Surgery, Oral Medicine, Oral Pathology, Oral Radiology and Endodontology 2005; 100/1: 125129.

13. Radha Krishnan S, Munshi A K, Hegde A M. Pulse oximetry: A diagnostic instrument in pulpal vitality testing. The Journal of Clinical Pediatric Dentistry 2002; 26/2: 141-145.

14. Khan R S, Gulabivala K, Snook M, Setchell D J. Evaluation of the pulse oximeter and the customized probe for pulp vitality testing. Journal of Endodontics 1996;22/3: 105-109.

15. Noblett W C, Wilcox L R, Scamman F, Johnson W T, Diaz-Arnold A. Detection of pulpal circulation in vitro by pulse oximetry. Journal of Endodontics 1996; 22/1: 1-5.

16. Vaghela, Dakshita Joy; Amit Sinha, Ashis. Pulp oximetry and Laser Doppler flowmetry for diagnosis of pulp vitality: Journal of interdisciplinary dentistry Jan-jun 2011/Vol 1/ issue 1.

17. Samraj RV. Recent advances in pulp vitality testing; Endodontology, Vol. 15, 2003.

18. S. Patel. New dimensions in endodontic imaging: part 2.Cone beam computed tomography; International Endodontic Journal: 2009.

19. Verdonschot, E. H., Wenzel, A. and Bronkhorst, E. M. Applicability of Receiver Operating Characteristic (ROC) analysis on discrete caries depth ratings. Community Dentistry and Oral Epidemiology, 21: 269-272. doi: 10.1111/j.1600-0528.1993.

20. E. Lynch and L. Abu-Naba'a, Dental Products News Journal, November 21, 2005.

21. Bennett T. Amaechi. Emerging technologies for diagnosis of dental caries: The road so far; J. Appl. Phys. 105, 2009: may

22. J. Yang and V. Dutra. Utility of radiology, laser fluorescence, and transillumination; Dent Clin North Am. 2005 October; 49(4): 739-52.

23. A. T. Stodt, Schweiz Monatsschr Zahnmed. Bitewing examinations as a part of preventive dentistry--a review; 114, 882 (2004).

24. D. A. Young. New caries detection technologies and modern caries management: merging the strategies;Gen Dent. 50, 320 (2002).

25. S. Keem and M. Elbaum. Wavelet representations for monitoring changes in teeth imaged with digital imaging fiber-optic transillumination. IEEE Trans Med Imaging 16, 653 (1997).

26. A. Schneiderman, M. Elbaum, T. Shultz, S. Keem, M. Greenebaum, and J.Driller. Assessment of dental caries with Digital Imaging Fiber-Optic TransIllumination (DIFOTI): in vitro study. Caries Res. 31, 103 (1997).

27. D. A. Young and J. D. Featherstone. Digital imaging fiber-optic trans-illumination, F-speed radiographic film and depth of approximal lesions. J Am Dent Assoc. 136, 1682 (2005)

28. D. N. Ricketts, E. A. Kidd, P. J. Liepins, and R. F. Wilson. Histological validation of electrical resistance measurements in the diagnosis of occlusal caries.Caries Res. 30, 148 (1996). 


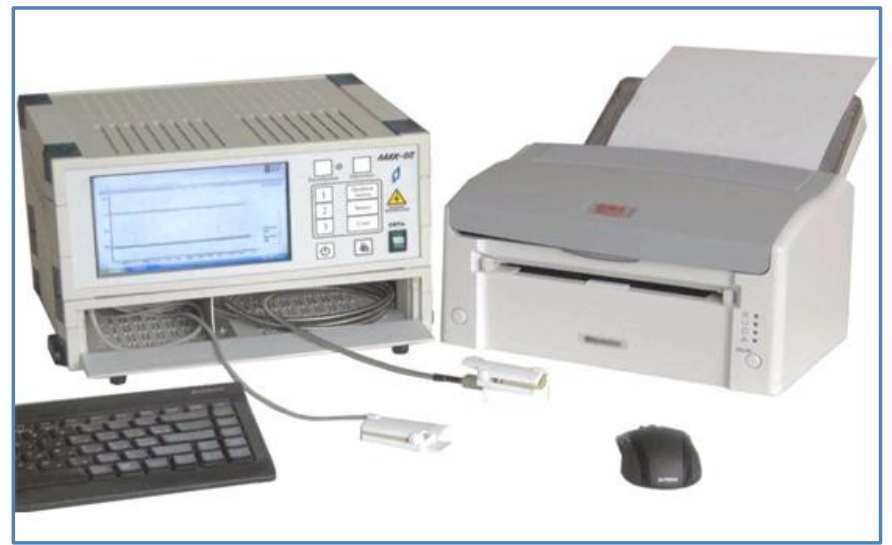

LEGEND 1: Fig. 1: Laser Doppler Flowmetry

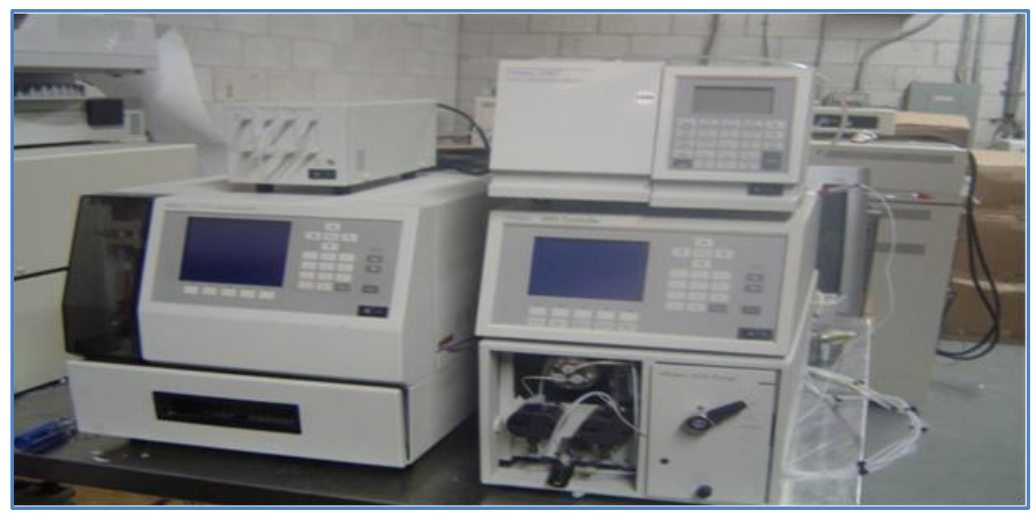

\section{LEGEND 2: Fig. 2: Dual Wave length Spectrophotometry}

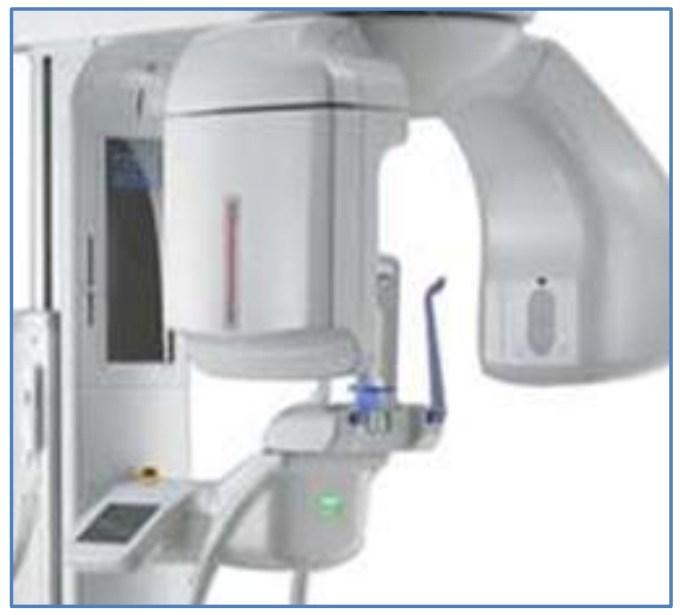

LEGEND 3: Fig. 3: Cone beam computed tomography 


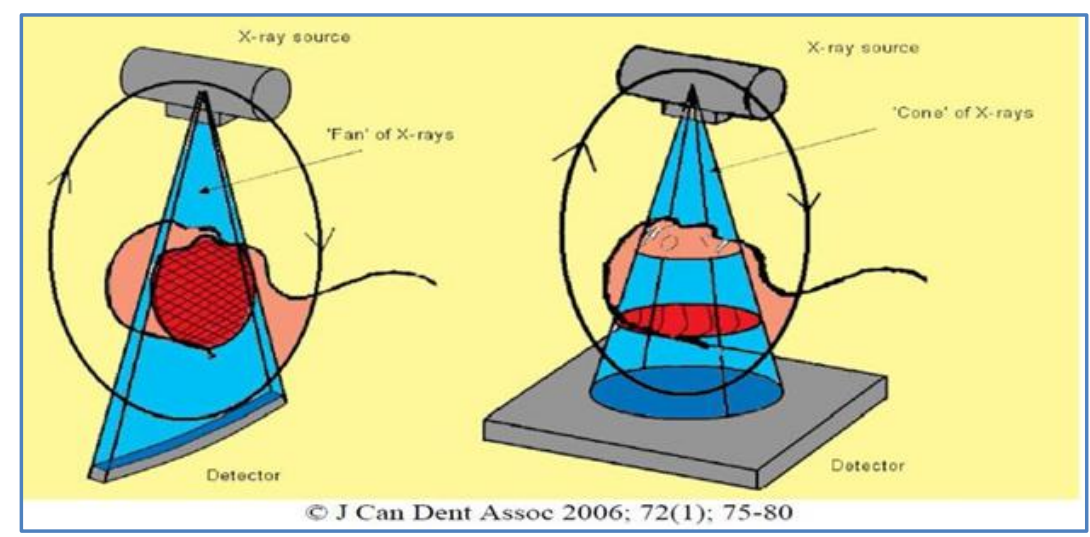

LEGEND 4: Fig. 4: Mechansim Of CBCT

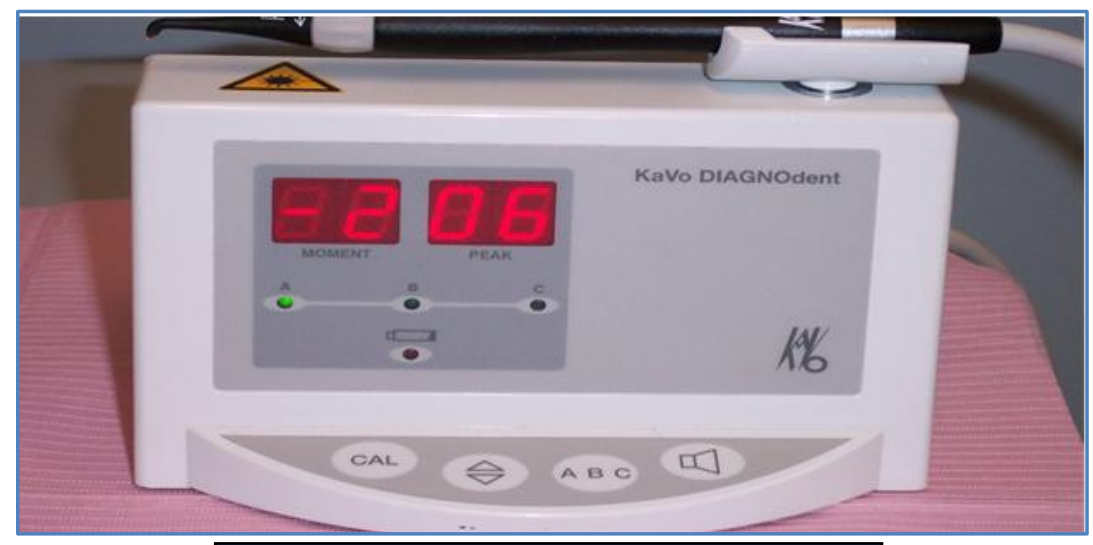

LEGEND 5: Fig. 5: Diagnodent Laser

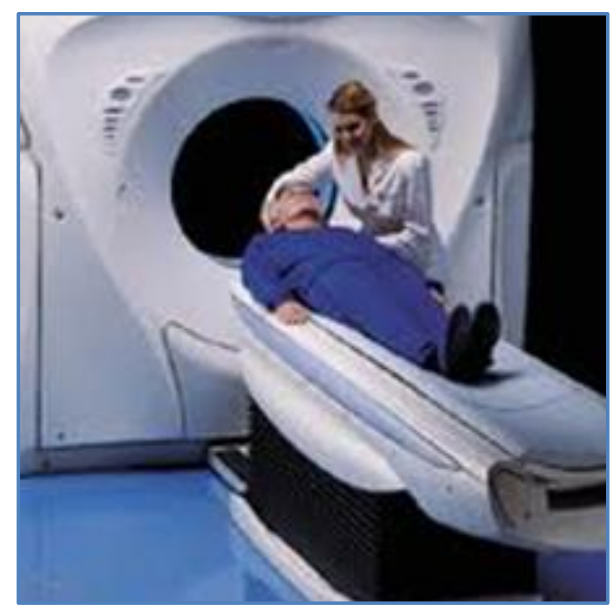

LEGEND 6: Fig. 6: Computed Tomography 


\section{AUTHORS:}

1. Divya Jindal

2. Deepak Raisingani

3. Medhavi Sharma

4. Dileep Soni

5. Hanisha Dabas

\section{PARTICULARS OF CONTRIBUTORS:}

1. Second Year Post Graduate, Department of Conservative Dentistry and Endodontics, Mahatma Gandhi Dental College and Hospital, Sitapura, Jaipur.

2. Professor and Head, Department of Conservative Dentistry and Endodontics, Mahatma Gandhi Dental College and Hospital, Sitapura, Jaipur.

3. Senior Lecturer, Department of Conservative Dentistry and Endodontics, Mahatma Gandhi Dental College and Hospital, Sitapura, Jaipur.

4. Second Year Post Graduate, Department of Conservative Dentistry and Endodontics, Mahatma Gandhi Dental College and Hospital, Sitapura, Jaipur.
5. First Year Post Graduate, Department of Conservative Dentistry and Endodontics, Mahatma Gandhi Dental College and Hospital, Sitapura, Jaipur.

\section{NAME ADDRESS EMAIL ID OF THE CORRESPONDING AUTHOR:}

Dr. Divya Jindal,

Second Year Post Graduate,

Department of Conservative Dentistry and

Endodontics,

Mahatma Gandhi Dental College and Hospital,

Sitapura (Jaipur) - 302022.

E-mail: jindaldivya89@gmail.com

Date of Submission: 25/12/2013.

Date of Peer Review: 26/12/2013.

Date of Acceptance: 21/01/2014.

Date of Publishing: 10/02/2014. 